\title{
Trismus caused by pterygomandibular raphe rigidity: A case report and systematic review
}

\author{
Kentaro Ouchi ${ }^{1,2 *}$, Yoko Ueno ${ }^{1}$, Hiroki Suzuki ${ }^{1,3}$ and Akio Jinnouchi ${ }^{1}$ \\ ${ }^{1}$ Department of Dentistry, Sasaguri Hospital, Inouekai Medical Corporation, Japan \\ ${ }^{2}$ Department of Dental Anesthesiology, Field of Maxillofacial Diagnostic and Surgical Sciences, Faculty of Dental Science, Kyushu University, Japan \\ ${ }^{3}$ Department of Implant and Rehabilitative Dentistry, Field of Oral Rehabilitation, Graduate School of Dental Science, Kyushu University, Japan
}

\begin{abstract}
Trismus is a well-known symptom in dental practice. Many of the causes are temporomandibular disorders, inflammation, tumor, and trauma. This report describes a case of trismus caused by pterygomandibular raphe rigidity. A systematic review was performed to identify reports on cause of trismus.

An 89-year-old woman complained of trismus and right mandibular displacement when mouth opening. In the intraoral examination, the rigidity of the right side pterygomandibular raphe was noted when mouth opening. The rigidity gingiva was not extensible, and it inhibited the rotational movement and forward movement of the right mandible, so it was considered that the mandible was displaced to the right during mouth opening. Therefore, for incision and mobilization operation of rigidity gingiva, patient was introduced to the Japanese Society of Oral and Maxillofacial Surgery (JSOMS) accreditation hospital. At the JSOMS accreditation hospital, an incision surgical operation was performed to transversely incise and longitudinally suture in the right side pterygomandibular raphe. The amount of mouth opening increased from $32 \mathrm{~mm}$ to $37 \mathrm{~mm}$ by an incision surgery.
\end{abstract}

This systematic review summarized the literature on the cause of trismus. TMJ disease was the most frequent disease-causing trismus. Then it was in order of tumor, inflammation, tetanus, scar and mandibular coronoid process hyperplasia. There was no report of pterygomandibular raphe rigidity. In this present case, the rigidity of pterygomandibular raphe was noted by detailed intraoral inspection. Therefore, it was considered that detailed intraoral inspection is necessary in dental medicine.

\section{Introduction}

Trismus is a well-known symptom in dental practice. Many of the causes are temporomandibular disorders, inflammation, tumor, and trauma $[1,2]$. There is no report of trismus caused by the rigidity of pterygomandibular raphe. We present a case of trismus caused by pterygomandibular raphe rigidity. A systematic review was performed to identify reports on cause of trismus.

\section{Case}

An 89-year-old woman (height $143 \mathrm{~cm}$, weight $37 \mathrm{~kg}$ ) complained of trismus and right mandibular displacement when mouth opening. She had a history of hypertension and mild dementia. She had been prescribed a course of amlodipine, spironolactone and furosemide for hypertension treatment. Dementia was 17 to 21 points in MMSE (Mini Mental State Examination), and a mild decline of cognitive function was observed.

The patient has complained of trismus 9 months ago also. At that time, with the MRI of the temporomandibular joint (TMJ), the joint disc was thin (Figure 1). Since then, she has been received a follow-up observation.

This time, she complained of trismus and right mandibular displacement when mouth opening. In the intraoral examination, the rigidity of the right side pterygomandibular raphe was noted when mouth opening (Figure 2). The rigidity gingiva was not extensible, and it inhibited the rotational movement and forward movement of the right mandible, so it considered that the mandible was displaced to the right during mouth opening. Therefore, for incision and mobilization operation of rigidity gingiva, patient was introduced to the Japanese Society of Oral and Maxillofacial Surgery (JSOMS) accreditation hospital.

At the JSOMS accreditation hospital, an incision surgical operation was performed to transversely incise and longitudinally suture (penetration of surgical suture is transversely) in the right side pterygomandibular raphe. By an incision surgery, the amount of mouth opening increased from $32 \mathrm{~mm}$ to $37 \mathrm{~mm}$, and the rightward displacement of the mandibular in mouth opening was also improved.

\section{Method}

This systematic review summarized the literature on the cause of trismus. A systematic review of all published literature was conducted to explore the cause of the trismus. Published literatures were identified by searching electronic databases. Databases were searched and

*Correspondence to: Kentaro Ouchi, DDS, PhD, Department of Dental Anesthesiology, Field of Maxillofacial Diagnostic and Surgical Sciences, Faculty of Dental Science, Kyushu University, 3-1-1 Maidashi, Higashi-ku, Fukuoka 812-8582, Japan, Tel: +81-92-641-1151, Fax: +81-92-642-6481; E-mail: ken2006anes@yahoo.co.jp

Key words: trismus, pterygomandibular raphe, rigidity

Received: September 15, 2018; Accepted: September 24, 2018; Published: September 28, 2018 


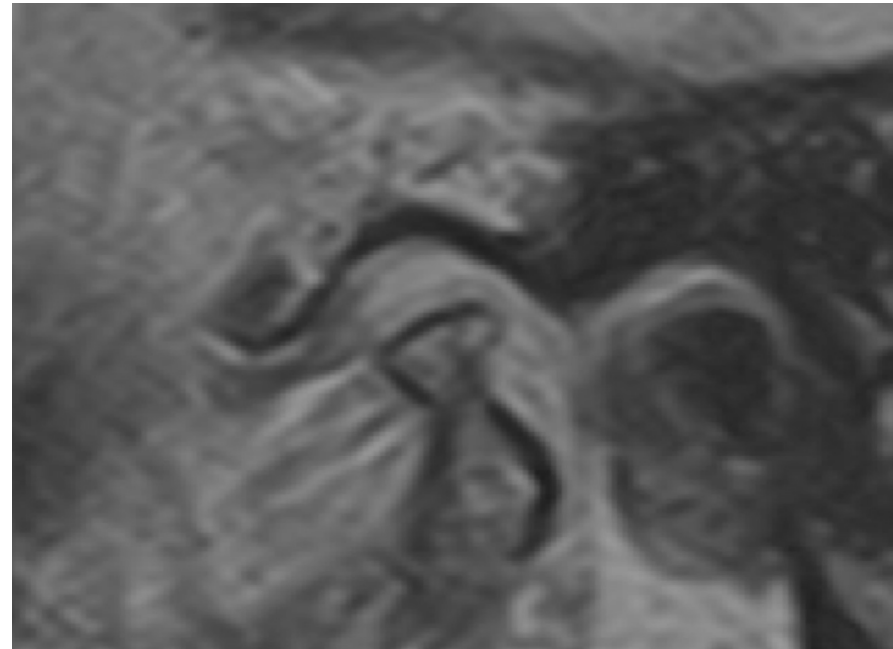

Figure 1. The MRI of the temporomandibular joint (TMJ) The joint disc was thin

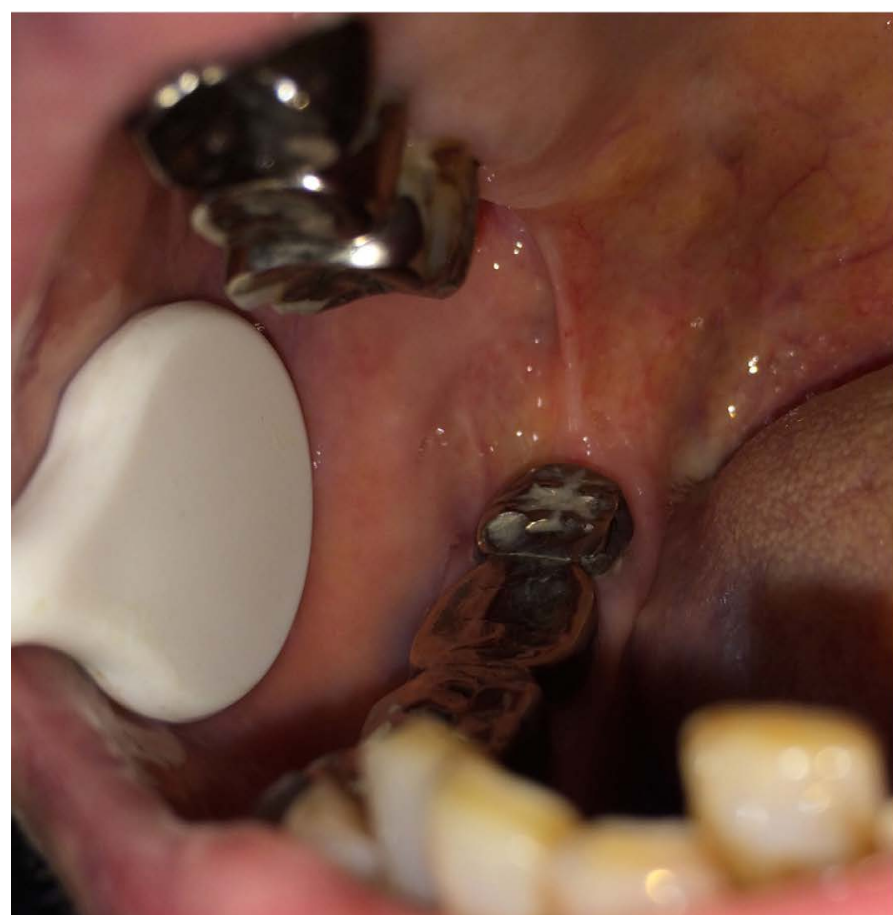

Figure 2. The right side pterygomandibular raphe. In the intraoral examination, the rigidity of the right side pterygomandibular raphe was noted when mouth opening

included: Pub Med (American National Library of Medicine) and Ichushi (Japan Medical Abstracts Society). Databases were searched in February 2017.

In Pub Med, the search formula was "(trismus [Title]) dental" and limited the article types to case reports. As a result, a total of 26 references were identified from the computerized search in Pub Med.

In Ichushi, the search formula was "((trismus/TH or trismus /AL)) and $((\mathrm{PT}=$ case reports $)$ and $(\mathrm{PT}=$ excluding conference presentation abstract) and SB=dental)". All search phrases were entered in Japanese. Attributes of search formula in Ichushi are shown below: (TH, include phrases in keyword; TA, Include phrases in title and abstract; PT, Article type; SB, Article classification). A total of 744 references were initially identified from the computerized search but 602 were excluded after screening the titles and article type. Exclusion criteria: commentary articles were excluded, and articles that did not include "trismus" in the title were excluded also. As a result, a total of 142 references were identified from the computerized search in Ichushi.

\section{Result}

In Pub Med, about the cause of trismus, each 4 cases were tumor and local anesthesia, which were the most frequent cause. Then it was masseter muscle atrophy, inflammation and scar (Figure 3).

In Ichushi, about the cause of trismus, 23 cases were TMJ disease, which was the most frequent cause. Then it was in order of tetanus, tumor, and inflammation (Figure 4). Some articles did not specify the cause of trismus, but most of them were case reports of anesthesia for patients with trismus $[3,4]$.

In the total of Ichushi and Pub Med, 25 cases with TMJ disease, 19 cases with tumor, 17 cases with inflammation, 16 cases with tetanus, 16 cases with scar, 16 cases with hyperplasia of mandibular coronoid process, 7 cases with masticatory muscle tendon-aponeurosis hyperplasia, 7 cases with congenital malformation, 6 cases with trauma, 5 cases with osseous malformation, 5 cases with masseter muscle atrophy, 5 cases with central nervous system disorder, 4 cases with local anesthesia, and other 1 to 2 cases each. There was no report of pterygomandibular raphe rigidity.

\section{Discussion}

Many of the causes of trismus are temporomandibular disorders, inflammation, tumor, and trauma [1,2]. There is no report of trismus caused by the rigidity of pterygomandibular raphe.

In this present case, the rigidity of the right side pterygomandibular raphe was noted when mouth opening. Accordingly, the right side pterygomandibular raphe was incised. Consequently, the amount of mouth opening increased from $32 \mathrm{~mm}$ to $37 \mathrm{~mm}$, and the rightward displacement of the mandibular in mouth opening was also improved.

This systematic review summarized the literature on the cause of trismus. About the cause of trismus, 25 cases were TMJ disease, which was the most frequent cause. Then it was in order of tumor, inflammation, tetanus, scar and mandibular coronoid process hyperplasia. There was no report of pterygomandibular raphe rigidity. In a rare report, there was a report of iatrogenic trismus that has adhered upper and lower teeth with glass ionomer cement [5]. There was also a report that trismus was caused by adhesion of upper and lower jaw teeth with a large amount of calculus [6].

In this present case, patient complained of trismus and right mandibular displacement when mouth opening. In the intraoral examination, the rigidity of the right side pterygomandibular raphe was noted when mouth opening. The rigidity gingiva was not extensible, and it inhibited the rotational movement and forward movement of the right mandible, so it was considered that the mandible was displaced to the right during mouth opening. Therefore, an incision surgical operation was performed to laterally incise and longitudinally suture in the right side pterygomandibular raphe. The amount of mouth opening increased by an incision surgery. In this present case, the cause of the rigidity only in the right side is not clear. It is known that oral mucosa increases fibrosis with age [7]. It was considered that fibrosis of the mucous membrane due to aging is involved. Although this patient has dementia, because dementia is mild, she was operable without general anesthesia/sedation. For patient with high-level dementia, intravenous anesthesia/sedation and inhalation sedation may be appropriate [8-10]. 

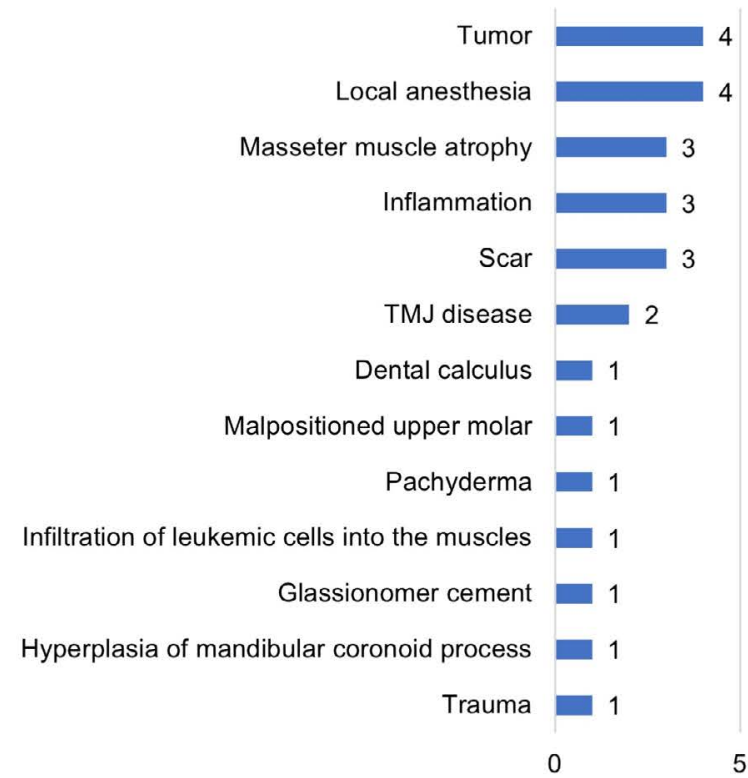

$5 \quad 10$

15

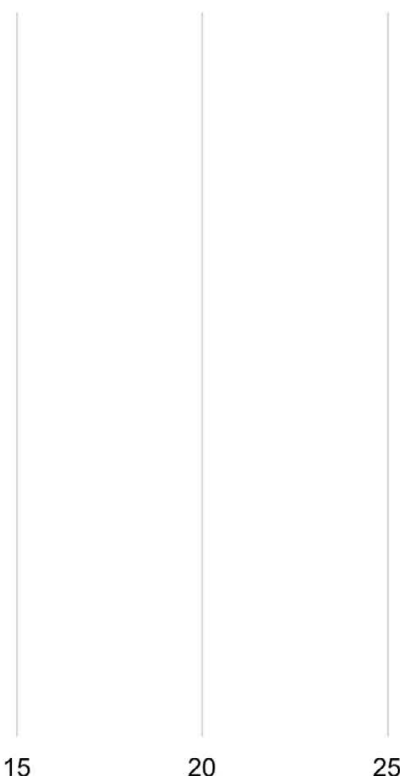

\section{Number of literature}

Figure 3. The cause of trismus in Pub Med

The search formula was "(trismus[Title]) dental", and limited the article types to case reports. As a result, a total of 26 references were identified from the computerized search in Pub Med

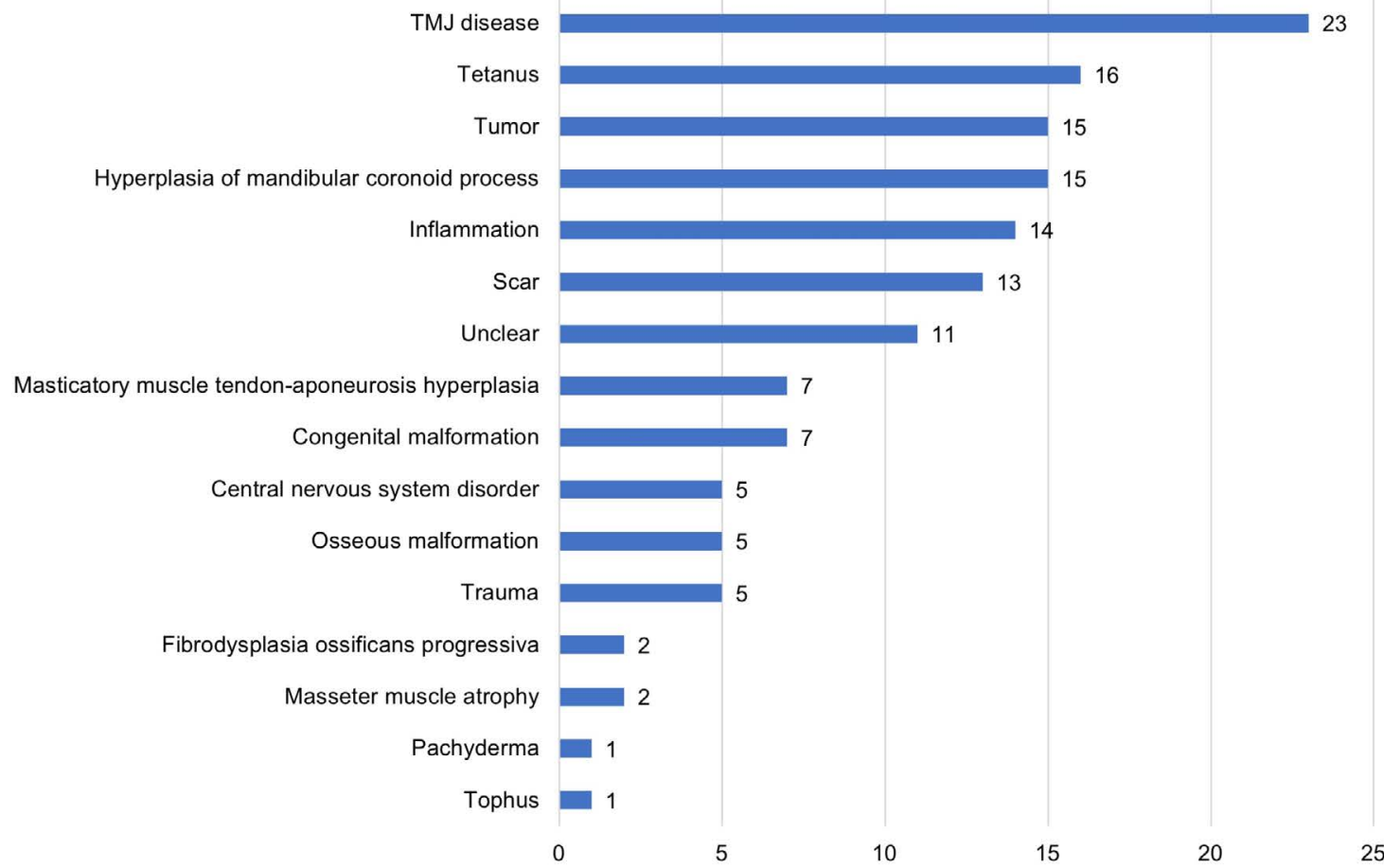

Number of literature

Figure 4. The cause of trismus in Ichushi

The search formula was "((trismus/TH or trismus $/ \mathrm{AL}))$ and $((\mathrm{PT}=$ case reports $)$ and $(\mathrm{PT}=$ excluding conference presentation abstract $)$ and $\mathrm{SB}=\mathrm{dental})$ ". $\mathrm{All}$ search phrases were entered in Japanese. Attributes of search formula in Ichushi are shown below: (TH, Include phrases in keyword; TA, Include phrases in title and abstract; PT, Article type; SB, Article classification). A total of 744 references were initially identified from the computerized search but 602 were excluded after screening the titles and article type. Exclusion criteria: commentary articles were excluded, and articles that did not include "trismus" in the title were excluded also. As a result, a total of 142 references were identified from the computerized search in Ichushi 
As a cause of trismus, pterygomandibular raphe rigidity is not widely known. Therefore, pterygomandibular raphe rigidity, which is usually unpredicted cause, can be overlooked. In this present case, the rigidity of pterygomandibular raphe was noted by detailed intraoral inspection. Therefore, it was considered that detailed intraoral inspection is necessary in dental practice.

\section{Conclusion}

This systematic review summarized the literature on the cause of trismus. TMJ disease was the most frequent disease-causing trismus. Then it was in order of tumor, inflammation, tetanus, scar and mandibular coronoid process hyperplasia. There was no report of pterygomandibular raphe rigidity. In this present case, the rigidity of pterygomandibular raphe was noted by detailed intraoral inspection. Therefore, it was considered that detailed intraoral inspection is necessary in dental practice.

\section{Acknowledgments}

This work was presented, in part, on June 15, 2017, at the 28th Annual Meeting of the Japanese Society of Gerodontology, Nagoya, Japan.

The authors thank Dr. Ami Maeno (Department of Oral and Maxillofacial Oncology, Field of Maxillofacial Diagnostic and Surgical Sciences, Faculty of Dental Science, Kyushu University; Chairman: Prof. Seiji Nakamura) for her help with oral surgery.

\section{References}

1. Luyk NH, Steinberg B (1990) Aetiology and diagnosis of clinically evident jaw trismus. Aust Dent J 35: 523-529. [Crossref]

2. Kato T, Mikami T, Funayama A, Niimi K, Tanaka R, et al, (2016) Bilateral hyperplasia of the mandibular coronoid process with remarkable trismus: a case report. Jpn J Jaw Deform 26: 214-219.

3. Kawabata K, Chida N, Yamauchi N, Yamakage M (2010) A case report of the successful nasotracheal intubation using airway scope and cuff inflation technique. J Jpn Dent Soc Anesthesiol 38: 311-312.

4. Akiyama M, Nago T, Yamada N, Fujii K, Sano K (2012) A case of intravenous sedation for patients with developmental disability and trismus. Journal of Japanese Society of Dentistry for Medically Compromised Patient 21: 141-146.

5. Basati M (2013) Cement-induced trismus. Br Dent J 215: 60. [Crossref]

6. Pillai K, Rawal Y (2001) Two cases of unusually heavy odontolithiasis--one causing trismus. Oral Surg Oral Med Oral Pathol Oral Radiol Endod 92: 365-366. [Crossref]

7. Akimoto K (2004) [Observations on the structural changes according to aging of ora mucous membrane in the elderly--structure of buccal mucous membrane in the vicinity of angulus oris. Kokubyo Gakkai Zasshi 71: 80-94. [Crossref]

8. Ouchi K (2017) A case of dental treatment with nitrous oxide for a patient with front temporal dementia. Japanese Journal of Gerodontology 32: 96-101.

9. Ouchi K, Sugiyama K (2015) Required propofol dose for anesthesia and time to emerge are affected by the use of antiepileptics: prospective cohort study. BMC Anesthesiol 15: 34. [Crossref]

10. Ouchi K, Fujiwara S, Sugiyama K (2017) Acoustic method respiratory rate monitoring is useful in patients under intravenous anesthesia. J Clin Monit Comput 31: 59-65. [Crossref]

Copyright: (C2018 Ouchi K. This is an open-access article distributed under the terms of the Creative Commons Attribution License, which permits unrestricted use, distribution, and reproduction in any medium, provided the original author and source are credited. 